\title{
Unexplained thrombosis of the aortic arch with distal embolization in a patient with altered fibrin clot properties
}

Tomasz Ciurus ${ }^{1}$, Anetta Undas², Malgorzata Lelonek ${ }^{1}$

1Department of Cardiology, Chair of Cardiology and Cardiac Surgery, Medical University of Lodz, Poland

2Institute of Cardiology, Jagiellonian University School of Medicine, Krakow, Poland

Submitted: 18 January 2011

Accepted: 26 July 2011

Arch Med Sci 2012; 8, 4: 733-735

DOI: 10.5114/aoms.2012.30298

Copyright @ 2012 Termedia \& Banach

The pathophysiology of arterial thrombosis in young patients remains unclear. Traditional cardiovascular risk factors explain not more than 50\% of cases of arterial thrombosis [1]. It is known that arterial inflammation, autoimmune diseases, endothelial dysfunction, atherosclerosis, blood flow abnormalities, and finally, altered platelet function, coagulation or fibrinolysis predispose to arterial thrombosis. The common thrombophilic factors, factor V Leiden and prothrombin G20210A polymorphism, are inconsistently associated with arterial thrombosis [2]. Growing evidence indicates that the structure of a fibrin clot composed of compact thin fibrin networks can predispose to arterial thrombosis [3]. However, a role of abnormal fibrin clot characteristics in arterial thrombosis different from coronary artery thrombosis is unknown. Aortic arch thrombosis (AAT) is a rare pathology and generally is associated with diffuse atherosclerosis of the aortic arch in elderly patients, aortic aneurysm or dissections [4]. However, mobile thrombi in the aortic arch have also been reported in younger patients [5].

We report here a young man who developed unexplained AAT and subsequent emboli to the lower limbs and, importantly, we detected novel prothrombotic fibrin alterations that may be behind this thrombosis.

A 35-year-old man, with no medical history (normal arterial, diabetes mellitus, lipid disorders, no smoking, normal weight) was admitted to the hospital due to paresis of the lower extremities. The patient was paraplegic; femoral and distal pulses were nonpalpable. Computed angiography showed an intraaortic mass which straddled the aortic bifurcation and extended to just below the renal arteries, indicative of a thrombus. Urgent embolectomy and fasciotomy in both lower extremities were performed.

An electrocardiogram showed a regular sinus rhythm at 70 beats/min, with no history of atrial fibrillation. Routine laboratory investigations did not show any abnormalities, with a normal value of blood lipids. C-reactive protein level was $2.82 \mathrm{mg} / \mathrm{l}$ and fibrinogen $4.11 \mathrm{~g} / \mathrm{l}$. Thrombophilia screening, including prothrombin gene 20210A mutation, factor V Leiden, lupus anticoagulant, antiphospholipid antibodies, protein C, free protein $\mathrm{S}$ and antithrombin, yielded negative results.

Interestingly, his mother underwent embolectomy of the aortic arch and brachiocephalic trunk, when she was 42 years.

In search for causes of arterial thrombosis one month after surgery, transoesophageal echocardiography was performed and revealed in the aortic arch a pedunculated mass of $2.6 \mathrm{~cm} \times 1.3 \mathrm{~cm}$, with no visible atherosclerotic plaques. Cardiac magnetic resonance imaging (MRI) showed

\author{
Corresponding author: \\ Assoc. Prof. Malgorzata \\ Lelonek MD, PhD, FESC \\ Department of Cardiology \\ Chair of Cardiology \\ and Cardiac Surgery \\ Medical University of Lodz \\ 1/3 Sterling \\ 91-425 Lodz, Poland \\ Phone/fax: +48 426364471 \\ E-mail: mlelonek@poczta.fm
}



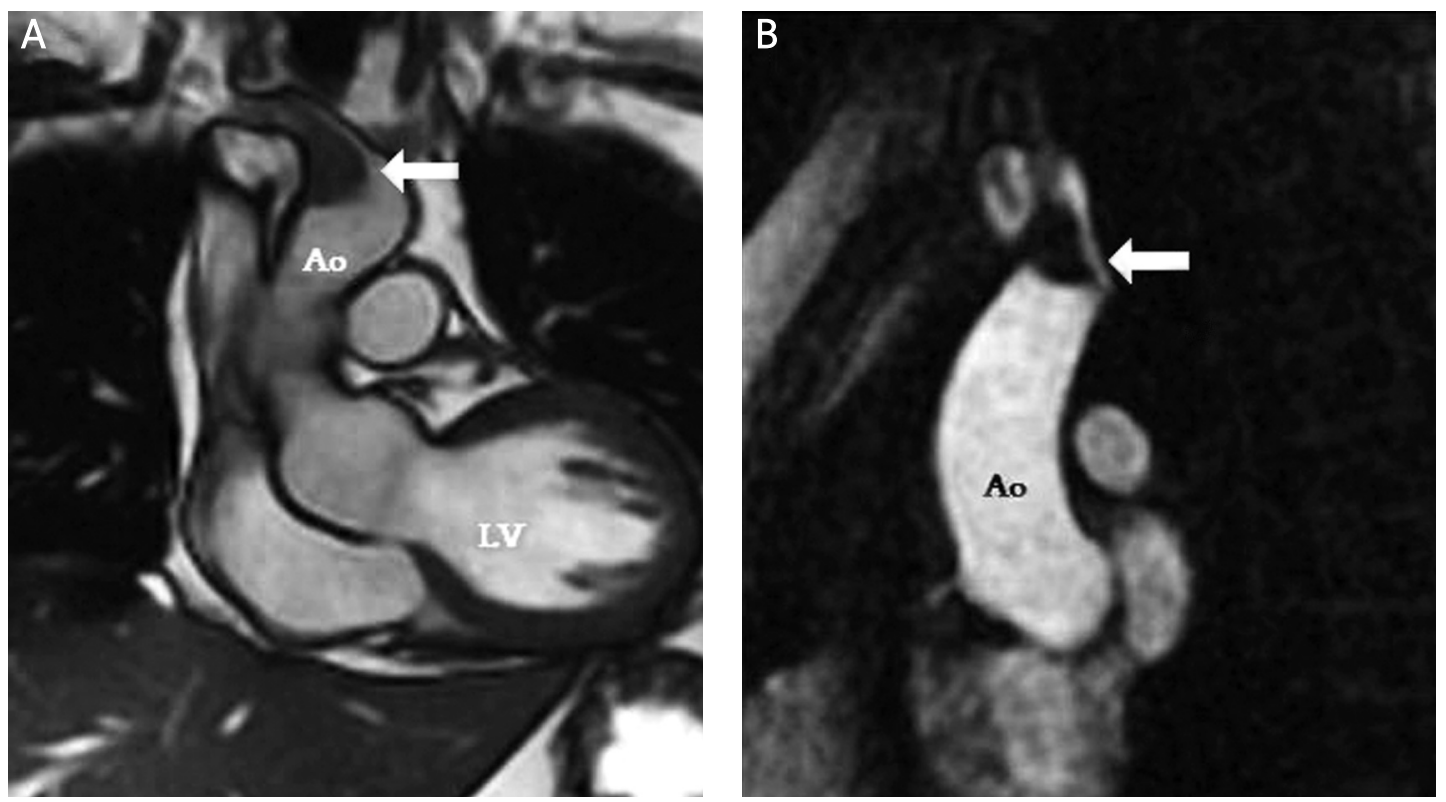

Figure 1. A mass attached to the aortic arch wall (arrow in the image) and closing brachiocephalic trunk ostium in MRI with a contrast injection (no amplification of the contrast in sequence of perfusion). A - a perfusion scan, $B$ - a cine image

Ao - aorta, $L V$ - left ventricle

a well-demarcated mass attached to the aortic arch wall and obstructing the brachiocephalic trunk ostium (Figure $1 \mathrm{~A}-\mathrm{B}$ ). There was no amplification of the contrast in the perfusion image of MRI, thus indicating the thrombus (Figure $1 \mathrm{~A}$ ).

The patient received intravenous unfractionated heparin followed by vitamin $\mathrm{K}$ antagonist (VKA) administration with a target international normalized ratio of 2-3 that resulted in a slight reduction in thrombus size within the first 3 weeks of therapy. At 12 months transoesophageal echocardiography, however, did not show any pathology in the aortic arch.

Given the data showing altered fibrin clot properties in patients with venous thromboembolism

Table I. Comparisons of fibrin clot features

\begin{tabular}{|lcc|}
\hline Feature & Patient & $\begin{array}{c}\text { Controls with INR } \\
\text { 2-3 }(n=20)\end{array}$ \\
\hline Ks $\left[10^{-9} \mathrm{~cm}^{2}\right]$ & 6.8 & $8.6 \pm 1$ \\
\hline Compaction [\%] & 49 & $63.8 \pm 6.2$ \\
\hline Lag phase $[\mathrm{s}]$ & 43 & $46.2 \pm 3.4$ \\
\hline$\Delta$ Abs $(405 \mathrm{~nm})$ & 0.89 & $0.72(0.68-0.8)$ \\
\hline$t_{50 \%}[\mathrm{~min}]$ & 9.7 & $7 \pm 1$ \\
\hline D-D max $[\mathrm{mg} / \mathrm{l}]$ & 3.98 & $3.1(2.6-3.6)$ \\
\hline D-D rate $[\mathrm{mg} / \mathrm{l} / \mathrm{min}]$ & 0.069 & $0.079(0.075-0.085)$ \\
\hline
\end{tabular}

Values are given as mean $\pm S D$. Ks indicates permeability coefficient, $\triangle A b s(405 \mathrm{~nm})$ - maximum absorbance of fibrin gel at $405 \mathrm{~nm}$ determined by using turbidimetry, $t_{50 \%}$ - half-lysis time, $D$ - $D$ max - maximum $D$-dimer levels in lysis assay 2, D-D rate-maximum rate of increase in D-dimer levels in lysis assay 2
[6] and ischemic stroke [7], we performed plasma fibrin structure/function analysis [6], which demonstrated markedly altered fibrin clot characteristics, including reduced clot permeability (a marker of pore size, Ks) and compaction (a marker of clot stiffness) combined with faster clot formation (shorter lag phase of fibrin formation) and impaired clot lysis (prolonged lysis time and slower rate of D-dimer release from clots) as compared to values obtained for anticoagulated patients with distal deep vein thrombosis (Table I).

Traditional cardiovascular risk factors explain arterial thrombosis in older patients [1], especially after interventions [8]. In young patients without cardiac risk factors, mostly arterial thrombotic events occurred related to atrial fibrillation or factor $\mathrm{V}$ Leiden. Thrombophilia screening fails to identify predisposing factors in $30 \%$ to $50 \%$ of idiopathic thrombosis patients [3]. A new risk factor for prothrombotic events revealed in venous thromboembolism, altered fibrin clot properties, is rarely described in patients with arterial thrombosis [3].

This study is the first to demonstrate AAT in a man with altered clot properties and reduced susceptibility to lysis. In the search for any cause of AAT in the patient with negative thrombophilia screening, abnormal features of plasma fibrin clot structure/function were detected. The propensity to faster formation of compact fibrin clots is associated with resistance to fibrinolysis, as evidenced by longer lysis time and lower lysis rate (Table I). By analogy to cryptogenic venous thrombosis [6] or stroke [7], such properties could also explain the 
occurrence of unexplained arterial thrombosis. Moreover, altered fibrin clot structure/function may be associated with an unusual location of thrombus formation.

Mechanisms underlying the fibrin clot characteristics in the current patient remain unclear and most likely combine environmental as well as genetic factors [3]. Although a positive familial history of AAT suggests a genetic background, we cannot definitely rule out in our patient other factors such as inflammatory, lipoprotein (a) (not measured in the present study) [9], or the effect of platelets and blood cells. An influence of other as yet unidentified genetic factors is also suggested.

It is unknown whether prolonged heparin administration, thrombolysis, high-intensity anticoagulant or a surgical excision is the best therapeutic option in such cases. Our study supports VKA therapy in AAT as shown in previous reports [3]. This approach is likely to prevent thrombus recurrence and its embolic complications. Our patient was also successfully treated with VKA since low thrombin activity during stable anticoagulation facilitates clot lysis.

In conclusion, the current report suggests that unfavorable fibrin clot features predispose to unexplained arterial thrombosis and embolism in young patients without thrombophilic factors, thus representing potential novel risk factors for thrombosis at untypical locations.

\section{Acknowledgments}

We would like to thank M. Maciejewski MD for echocardiographic images. The study was given approval by the ethics committee of the Medical University of Lodz and informed consent was given by the subject.

\section{References}

1. Kullo IJ, Gau GT, Tajik AJ. Novel risk factors for atherosclerosis. Mayo Clin Proc 2000; 75: 369-80.

2. Reitsma PH, Rosendaal FR. Past and future of genetic research in thrombosis. J Thromb Haemost 2007; 5 (Suppl 1): 264-9.

3. Scott EM, Ariens RAS, Grant PJ. Genetic and environmental determinants of fibrin structure and function: relevance to clinical disease. Arterioscler Thromb Vasc Biol 2004; 24: 1558-66.

4. Laperche T, Laurian C, Roudaut R, Steg PG. Mobile thromboses of the aortic arch without aortic debris. A transesophageal echocardiographic finding associated with unexplained arterial embolism. Circulation 1997; 96: 288-94.

5. Choukroun EM, Labrousse LM, Madonna FP, Deville C. Mobile thrombus of the thoracic aorta: diagnosis and treatment in 9 cases. Ann Vasc Surg 2002; 16: 714-22.

6. Undas A, Zawilska K, Ciesla-Dul M, et al. Altered clot structure/function in patients with idiopathic venous thromboembolism and in their relatives. Blood 2009; 114: 4272-8.
7. Undas A, Podolec P, Zawilska K, et al. Altered fibrin clot structure/function as a novel risk factor for cryptogenic ischemic stroke. Stroke 2009; 40: 1499-501.

8. Kaminski KA, Knap M, Werel D, Musial WJ. Aortic thrombosis as a complication of intraaortic ballon. Arch Med Sci 2009; 4: 640-3.

9. Undas A, Stepien E, Tracz W, Szczeklik A. Lipoprotein(a) as a modifier of fibrin clot permeability and susceptibility to lysis. J Thromb Haemost 2006; 4: 973-5. 